[0212-7199 (2003) 20: 6; pp 297-300] ANALES DE MEDICINA INTERNA Copyright @ 2003 ARAN EDICIONES, S.L.

AN. MED. INTERNA (Madrid) Vol. 20, N. ${ }^{\circ} 6$, pp. 297-300, 2003

\section{Adecuación de los ingresos y estancias en un Servicio de Medicina Interna de un hospital de segundo nivel utilizando la versión concurrente del AEP (Appropriateness Evaluation Protocol)}

\author{
F. J. RODRÍGUEZ-VERA, Y. MARÍN FERNÁNDEZ, A. SÁNCHEZ, \\ C. BORRACHERO, E. PUJOL DE LA LLAVE
}

Servicio de Medicina Interna. Hospital Juan Ramón Jiménez. Huelva

APPROPRIATENESS OF THE ADMISSIONS AND STAYS IN AN INTERNAL MEDICINE DEPARTMENT OF A SECONDARY HOSPITAL USING THE CONCURRENT VERSION OF THE AEP (APPROPRIATENESS EVALUATION PROTOCOL)

\section{RESUMEN}

Objetivos: Determinar la inadecuación de ingresos y estancias en un Servicio de Medicina Interna utilizando la versión concurrente del AEP (Appropriateness Evaluation Protocol).

Método: Aplicación de la versión concurrente del AEP a los pacientes ingresados en un Servicio de Medicina Interna.

Resultados: 59 pacientes y 485 estancias fueron evaluados. Un $15.3 \%$ de los ingresos y un $33 \%$ de las estancias fue inadecuado. La causa más frecuente de ingreso inadecuado fue agilización del estudio ambulatorio. El motivo más frecuente de estancia inadecuada fue la espera de resultados de pruebas complementarias e interconsultas.

Conclusiones: La utilización de la versión concurrente del AEP permite obtener de forma cómoda y rápida información del sobreuso de los recursos de hospitalización. Los resultados obtenidos son similares a los de otros estudios realizados de forma retrospectiva en España.

PALABRAS CLAVE: Appropriateness Evaluation Protocol. Adecuación de ingresos. Adecuación de estancias. Evaluación concurrente.
ABSTRACT

Aims: To Study the inappropriateness of the admissions and stays in an Internal Medicine Department using the concurrent version of the AEP (Appropriateness Evaluation Protocol).

Method: Aplication of the concurrent version of the AEP to all the patients internated in an Internal Medicine Department.

Results: 59 patients and 485 stays fulfilled the inclusion criteria. A $15.3 \%$ of the admissions and a 33\% of the stays did not acomplish the criteria of appropriateness of the AEP. The most frequent cause of innappropriate admision was to avoid the delay of the ambulatory studies. The most common cause of innappropriate stay was the wait for results of complementary tests and consultancy between different specialities.

Conclusions: Using the concurrent version of the AEP allows to obtain information about the overuse of the hospitalization resources in an easy and rapid way. The results obtained in our study are similar to other made in Spain using the retrospective version of the AEP.

KEY WORDS: Appropriateness. Evaluation Protocol. Appropriateness of admissions. Appropriateness of stay. Concurrent Evaluation.

Rodríguez-Vera FJ, Marín Fernández Y, Sánchez A, Borrachero C, Pujol de la Llave E. Adecuación de los ingresos y estancias en un Servicio de Medicina Interna de un Hospital de segundo nivel utilizando la versión concurrente del AEP (Appropriateness Evaluation Protocol). An Med Interna (Madrid) 2003; 20: 297-300.

\section{INTRODUCCIÓN}

La utilización de herramientas para determinar la adecuación de utilización de los recursos sanitarios, entre los que se encuentran las camas de hospitalización, ha sido un tema debatido frecuentemente en la literatura médica (1-6). En nuestro medio, el instrumento de medida más utilizado para determinar el sobreuso de los recursos de hospitalización es el AEP (Appropriateness Evaluation Protocol), que consiste en criterios explícitos que evalúan un ingreso o estancia como adecuado si cumple al menos uno de ellos (7). En España se han realizado varios estudios sobre adecuación de ingresos (8-14), factores asociados a la inadecuación (1519) e incluso se han llevado a cabo planes de actuación basados en los datos obtenidos en estudios realizados (20). Uno de los más importantes inconvenientes del AEP consiste en la necesidad de realizar revisiones retrospectivas de gran número de historias clínicas, lo que hace difícilmente realizable este control de forma periódica y no aporta información rápida acerca del sobreuso de la hospitalización, que puede resultar de gran utilidad para elaborar planes de actuación en épocas de alta frecuentación hospitalaria. Para una evaluación rápida, el manual del usuario del AEP propone la utilización concurrente (trasversal) del protocolo. Aunque en Europa ya se han realizado estudios utilizando esta versión (21), no se hemos encontrado en España ningún trabajo que la contemple. En el presente estudio nos proponemos evaluar la inadecuación de los ingresos y estancias en una sección de Medicina Interna utilizando la versión concurrente del AEP. 


\section{MATERIAL Y MÉTODO}

El Hospital Juan Ramón Jiménez de Huelva es un centro hospitalario de segundo nivel con una capacidad de 600 camas, de las que 64 pertenecen al Servicio de Medicina Interna, y es centro de referencia para toda la provincia de Huelva. En este estudio fueron evaluadas las historias clínicas de todos los pacientes que se encontraban ingresados en camas de Medicina Interna y los asignados a esta especialidad en camas no pertenecientes al servicio el día 27 de febrero de 2002, excluyendo aquellos que habían ingresado ese mismo día y los enfermos de otras especialidades ingresados en camas de Medicina Interna. Dos revisores no responsables del ingreso ni la estancia hospitalaria revisaron independientemente cada una de las historias clínicas, encontrándose a su disposición un tercer evaluador experimentado en revisión de historias clínicas y aplicación de AEP en caso de discordancia. Se revisaron parámetros demográficos (edad y género), clínicos (multingresador -todo paciente que ha ingresado más de dos veces en el ultimo año o tres en los ultimo cinco años-, patología que motivó el ingreso), estructurales (franja horaria de ingreso mañana, tarde, noche-, tipo de ingreso -urgente o programado- y tiempo de transcurrido desde su llegada al hospital hasta la formalización de la ficha de ingreso), parámetros de gestión (estancia media en el momento del estudio, pacientes hospitalizados fuera de las camas asignadas al servicio) y estudio de adecuación del ingreso y estancia hospitalaria. Los datos obtenidos fueron introducidos en una base de datos Access y analizados con el programa estadístico SPSS 10,0 para Windows, determinándose medias e intervalos de confianza del $95 \%$.

\section{RESULTADOS}

Cincuenta y nueve pacientes, que generaron 485 estancias, cumplieron los criterios de inclusión en el estudio. 17 (28,81\%) se encontraban ingresados fuera de la Unidad de Medicina Interna. La edad media fue de 65,85 a (IC $\pm 12,54$ ). El 62,7\% de los pacientes estudiados era de género femenino y el $37,3 \%$ eran varones. Un 39\% reunía criterios de multingreso. Por grupos de patología, las enfermedades cardiovasculares fueron la causa más frecuente de ingreso $(49,2 \%$ del total), seguida por patología infecciosa $(11,9 \%)$. El 72,9\% de los pacientes ingresó en el tramo horario de tarde (15-24 horas), mientras que sólo un $8,5 \%$ lo hizo en la jornada laboral. Un $86,4 \%$ accedió a nuestros servicios de hospitalización desde Urgencias, mientras que el $13,6 \%$ restante lo hizo de forma reglada, desde consultas. Sólo un $15,3 \%$ de los pacientes esperó menos de 5 horas desde la llegada hasta el ingreso, el 47,3\% había esperado entre 5 y 12 horas y el 37,4\% más de 12 horas. La estancia media de los pacientes evaluados fue de 8,22 días (IC $+/-8,40$ ), con una media de 2,17 días de estancia inadecuada (IC +/- 3,29).

Encontramos un $15,3 \%$ de ingresos que no reunía ningún criterio de adecuación. La causa más frecuente de adecuación del ingreso fue la necesidad de medicación intravenosa y/o reposición de fluidos, seguido por la necesidad de ventilación asistida o intermitente (Tabla I). El motivo más frecuente de inadecuación del ingreso fue para agilizar el estudio ambulatorio $(66,6 \%)$. Otros motivos de inadecuación fueron pruebas diagnósticas que podrían haberse realizado en consultas externas y la necesidad de institucionalización del paciente pero en un nivel asistencial menos agresivo.

El $67 \%$ de las estancias evaluadas cumplió criterios de
TABLA I

\begin{tabular}{lc}
\multicolumn{2}{c}{ TABLA I } \\
\hline CAUSAS DE ADECUACIÓ N DE INGRESO S \\
\hline Causas & Porcentaje \\
\hline Necesidad de medicación intravenosa & $45,88 \%$ \\
Ventilación asistida continúa o intermitente & $12,94 \%$ \\
Electrocardiograma compatible con isquemia aguda & $9,4 \%$ \\
Alteración electrolítica o ácido-base severa & $5,88 \%$ \\
Pérdida brusca de movilidad & $4,7 \%$ \\
Fiebre persistente más de 5 días & $4,7 \%$ \\
Cirugía o técnica especial & $3,5 \%$ \\
Alteraciones de la frecuencia cardíaca & $5,88 \%$ \\
Estado confusional agudo,coma o falta de respuesta & $2,27 \%$
\end{tabular}

adecuación. La causa que más frecuentemente justificó la estancia en el servicio fue la necesidad de terapia parenteral intermitente o continua, seguida de la necesidad de terapia respiratoria e inyecciones intramusculares o subcutáneas. Con menor frecuencia se encontraron la realización de procedimientos invasivos durante las últimas 24 horas, dificultades neurológicas y necesidad de control de balances (Tabla II). Un $73 \%$ de las estancias adecuadas satisfacía dos o más criterios de adecuación de la estancia.

La causa más frecuente de inadecuación de la estancia fue la espera de resultados de pruebas e interconsultas, seguida de días de no trabajo y de problemas de calendario para realizar pruebas diagnósticas (Tabla III). En cuanto al número de criterios de inadecuación de la estancia, el 84,6\% cumplía dos o más criterios de inadecuación de la estancia.

\section{DISCUSIÓN}

La utilización de la versión concurrente del AEP nos indica de forma instantánea la situación de sobreuso de los recursos de hospitalización, y es el modo más rápido de elaborar estrategias encaminadas a hacer el sistema más eficiente, ofertando una mayor calidad asistencial sin incremento del coste económico. Los datos aportados por el estudio nos muestran un grupo poblacional importante (casi el 40\%) que está constituido por pacientes reingresadores. El hecho de que sólo un $15 \%$ de los pacientes ingresara antes de las 5 horas de su llegada al hospital nos hace pensar en la posibilidad de que un número importante de pacientes precisara estabilización previa en el área de Urgencias debido a inestabilidad clínica, hecho que junto a la elevada incidencia de patología cardiovascular y bajo número de ingresos por alteraciones electrocardiográficas hace pensar en la posibilidad de patología crónica y nos hace plantearnos la necesidad de un estudio más exhaustivo acerca de los motivos que originan el ingreso hospitalario repetido y la puesta en marcha de medidas con vistas a minimizarlo.

Casi un $73 \%$ de los pacientes ingresó en la franja horaria de tarde (15-24 horas), tiempo en el que el ingreso corre a cargo del internista de guardia, que no es necesariamente un especialista en Medicina Interna de nuestro servicio. Teniendo en cuenta el hecho de que un elevado porcentaje de pacientes permaneció en el Servicio de Urgencias más de 5 horas, pensamos que quizá el proceso sería optimizable realizando una evaluación y orientación clínica inicial en el horario laboral por un 
TABLA II

CAUSAS DE ADECUACIÓN DE ESTANCIAS

\begin{tabular}{lc}
\hline Causas & Porcentaje \\
\hline Necesidad terapia parenteral intermitente o continua & $35,6 \%$ \\
Necesidad de terapia respiratoria & $15,84 \%$ \\
Necesidad de medicación intramuscular o subcutánea & $15,84 \%$ \\
Procedimientos invasivos en las últimas 24 horas & $5,9 \%$ \\
Dificultades neurológicas & $3,9 \%$ \\
Necesidad de control de balances & $3,9 \%$ \\
Prueba con control dietético estricto & $1,98 \%$ \\
Tratamiento nuevos que requieren frecuentes & $1,98 \%$ \\
$\quad$ ajustes de dosis & $1,98 \%$ \\
Intervención quirúrgica en 24 horas & $1,98 \%$ \\
Estado confusional agudo & $1,98 \%$ \\
M onitorización por enfermera con supervisión médica & \\
$\quad 3$ veces/día & $0,99 \%$ \\
Intervención quirúrgica/ cateterización cardiaca ese día & $0,99 \%$ \\
Dificultades neurológicas & $0,99 \%$ \\
Cuidado de heridas quirúrgicas mayores y drenajes & $0,99 \%$ \\
Necesidad de transfusión por pérdida sanguínea & \\
24 horas antes & \\
\hline
\end{tabular}

TABLA III

\begin{tabular}{lc}
\multicolumn{2}{c}{ CAUSAS DE INADECUACIÓ N DE ESTANCIAS } \\
\hline Causas & Porcentaje \\
\hline Espera de resultados de pruebas e interconsultas & $33,89 \%$ \\
Días de no trabajo del personal médico & $22,03 \%$ \\
$\begin{array}{l}\text { Problemas de calendario para realización de } \\
\quad \text { pruebas diagnósticas }\end{array}$ & $13,55 \%$ \\
$\begin{array}{l}\text { Posibilidad de realización de procedimientos diagnósticos } \\
\quad \text { y terapéuticos de forma ambulatoria }\end{array}$ & $10,16 \%$ \\
$\begin{array}{l}\text { Problema de calendario para intervención quirúrgica } \\
\text { Falta de disponibilidad de instalaciones alternativas }\end{array}$ & $1,69 \%$ \\
$\begin{array}{l}\text { Falta de disponibilidad de dispositivos de } \\
\quad \text { atención domiciliaria }\end{array}$ & $1,69 \%$ \\
\hline
\end{tabular}

Internista perteneciente al servicio donde ingresó el paciente.

El ingreso programado desde consultas, superior al obtenido en otros estudios $(11,13)$, probablemente quedó destinado a aquellos pacientes que se encontraban en estudio y el clínico sospechaba la posibilidad de una patología grave que no permitía la demora que supone un estudio reglado desde consultas. En este aspecto, es posible que la aplicación de alguna medida de priorización a los estudios ambulatorios permitieran evitar estos ingresos. Además de aquellos, la presencia de enfermedades mal definidas, que se definen como síndromes generales que se ingresan para estudio puede ser otro motivo de ingreso desde consultas.

Los pacientes ingresados fuera de las camas pertenecientes al Servicio de Medicina Interna (pacientes periféricos) supusieron casi un tercio del total de los evaluados. Tal circunstan- cia puede mostrar en alguna medida la carga asistencial del Servicio, así como su elasticidad para adaptarse a circunstancias extraordinarias. Este hallazgo nos ha llevado a plantearnos la posibilidad de estudiar este grupo poblacional con objeto de determinar si el internamiento fuera de las camas asignadas a la especialidad incide de alguna forma en alargamiento de la estancia, mayor inadecuación de la misma, o menor número de cuidados que el resto de los pacientes, producidos por distancia física del médico responsable y falta de familiaridad del personal sanitario de la planta correspondiente con los procedimientos empleados en Medicina Interna.

La estancia media de nuestros pacientes fue de 8,22 días. Teniendo en cuenta la exclusión del estudio de pacientes ingresados ese mismo día, esta cifra puede reflejar de forma adecuada la estancia media real de nuestros pacientes. La detección de la existencia de 2,17 días de estancia inadecuada según los criterios del AEP, nos muestra la potencialidad de mejora de esta cifra.

En el presente estudio hemos detectado cifras de inadecuación del ingreso y estancia similares a las de otros estudios llevados a cabo $(11,22)$. Los porcentajes de inadecuación de ingresos y estancias coinciden con los resultados de otros estudios llevados a cabo (22-24), así como los motivos que los justifican. La causa más frecuente de inadecuación del ingreso fue la agilización del estudio ambulatorio. Pensamos que una mayor rapidez en la realización de las pruebas complementarias solicitadas desde consultas, así como una atención rápida desde el momento de la petición de consulta en Medicina Interna, pueden contribuir a reducir el número de ingresos originados por este motivo. Con este objetivo, fue creada recientemente en nuestro centro la consulta rápida de Medicina Interna (CRMI), donde además de remitirse a los pacientes que se encuentran en la situación anteriormente descrita, se atiende de forma prioritaria (retraso medio de una semana) a personas remitidas desde el Servicio de Urgencias de nuestro Centro que podría haber ingresado por demora del estudio ambulatorio para el estudio de su patología.

La espera de resultados de pruebas diagnósticas, descrito en el manual del usuario del AEP como el tiempo transcurrido desde que la prueba o consulta ha sido solicitada hasta que el informe correspondiente se encuentra en la historia clínica (25) fueron la causa de la mayor parte de días de estancia inadecuados. Estas estancias inadecuadas pudieran ser evitadas remitiendo a una consulta de Medicina Interna en un período de tiempo razonable a los pacientes sin criterios de gravedad cuyo único motivo de ingreso es la espera de resultados. En este aspecto la CRMI también desempeña un papel importante, recibiendo todos los pacientes que cumplen estas características y atendiéndoles en el plazo de tiempo que su médico responsable considere oportuno.

Los días de no trabajo supusieron la segunda causa de inadecuación de la estancia hospitalaria. Suponen estos una serie de jornadas en las que el paciente recibe cuidados de enfermería, que sólo en ocasiones justifican per se, la estancia, pero no es objeto de ninguna atención específica por parte del clínico ni de los servicios centrales. Una posible solución a este problema consistiría en destinar personal a la atención clínica y servicios centrales los días no laborables.

Otro motivo de inadecuación de la estancia que llamó poderosamente nuestra atención fue la posibilidad de haber llevado a cabo el manejo del paciente como externo. En muchas ocasiones, la existencia de retrasos en las listas de espera de las consultas externas supone un efecto disuasorio 
para continuar el proceso de diagnóstico o tratamiento del paciente de forma ambulatoria. En ese mismo aspecto incide también la CRMI, con la posibilidad de remisión directa en la fecha estimada oportuna por el facultativo responsable del paciente, quien al mismo tiempo establece en el informe de alta hospitalaria la actitud recomendable a seguir.

Los criterios de adecuación de la estancia hospitalaria también merecen una serie de reflexiones. A diferencia de otros países (21,26-31), en España no disponemos de versión del AEP adaptada a nuestro entorno, de forma que los criterios originalmente ideados por Restuccia (25) pueden no adaptarse completamente a nuestra realidad asistencial. El principal motivo de adecuación es paradigma de ello: la necesidad de medicación parenteral no supone en algunas versiones adaptadas en Europa (21) un criterio de adecuación de ingreso y estancia. Tal medicación, plantean las versiones adaptadas, puede ser administrada de forma ambulatoria por un servicio de asistencia domiciliaria, o bien en el propio hospital, dentro del hospital de día. La variabilidad de la práctica clínica también supone una causa de variabilidad en la adecuación de ingresos y estancias. El segundo motivo de adecuación del ingreso (necesidad de terapia respiratoria, dentro de la que el manual incluye el uso de nebulizaciones) es el paradigma de ello, en cuanto que puede encontrarse prescrito este tipo de terapia en casos en que no resulta estrictamente necesario en pacientes que se encuentran estables desde el punto de vista gasométrico.

Para finalizar, el estudio demuestra que la utilización concurrente del AEP en un servicio o sección puede ser una herramienta cómoda y útil para detectar problemas o deficiencias en el correcto funcionamiento del sistema en un momento determinado, pudiendo servir como instrumento para la elaboración de planes destinados a solucionarlos y medir el impacto de las medidas tomadas a través de una nueva aplicación concurrente del protocolo cuando se estime oportuno.

\section{Bibliografía}

1. Peiró Moreno S, Portella E. Identificación del uso inapropiado de la hospitalización: la búsqueda de la eficiencia. Med Clin (Barc) 1994; 103: 65-71.

2. McDonagh MS, Smith DH, Goddard M. Measuring appropriate use of acute beds. A systematic review of methods and results. Health Policy 2000; 53: 157-84.

3. Restuccia JD. The evolution of hospital utilization review methods in the United States. Int J Qual Health Care 1995 ; 7: 253-60.

4. Donald IP, Jay T, Linsell J, Foy C. Defining the appropriate use of community hospital beds. Br J Gen Pract 2001; 51: 95-100.

5. Ollero Baturone M. Adecuación y utilidad del ingreso hospitalario. Med Clin (Barc) 2001; 116: 655-7.

6. Callejas-Rubio JL, Fernández-Moyano A, Palmero C, Navarro MD. ¿Son realmente objetivos los criterios de adecuación hospitalario del Appropriateness Evaluation Protocol (AEP)?. Med Clin (Barc) 2002; 118 (4): 157-8.

7. Gertman PM, Restuccia JD. The Appropriateness Evaluation Protocol: a technique for asessing unnecessary days of hospital care. Med Care 1981; 19: 855-71.

8. Bañeres J, Alonso J, Broquetas J, Antó J. Ingresos hospitalarios inadecuados y días de estancia inactivos en pacientes con enfermedad obstructiva crónica y neoplasia pulmonar. Med Clin (Barc) 1993; 100: 407-11.

9. Matorras Galan P, de Pablo Casas M, Otero Garcia L, Alonso Lopez F, Daroca, Perez R, et al. Adecuación de los ingresos en un servicio de medicina interna de un hospital de tercer nivel. Med Clin (Barc) 1990; 94: $290-3$.

10. Lorenzo S, Sunol R. An overview of Spanish studies on appropriateness of hospital use. Int J Qual Health Care 1995; 7: 213-8.

11. Rodríguez-Vera FJ, Alcoucer Díaz MR, Rodríguez Gómez FJ, Camacho Martínez T, Colchero Fernández J, Pujol de la Llave E. Adecuación de los ingresos en un Servicio de Medicina interna de un Hospital de segundo nivel. An Med Interna (Madrid) 1999; 16: 277-80.

12. Ochoa-Gómez J, Villar Arias A, Ramalle-Gómara E, Carpintero Escudero JM, Bragado Blas L, Ruiz Aspiazu JI. Adecuación de los ingresos hospitalarios urgentes. An Med Interna (Madrid) 2002;19: 446-8.

13. Tomás Vecina S, Duaso Magaña E, Ferrer Tarres JM, Rodríguez Caballeira M, Porta Castejón R, Epelde Gonzalo F. Evaluación del uso apropiado de un area de observación de urgencias mediante el Appropriateness Evaluation Protocol; un análisis de 4.700 casos. An Med Interna (Madrid) 2000; 17: 229-37.

14. Miron Canelo JA, Orosco Pedemonte JA, Alonso Sardon M, Saenz Gonzalez MC. Estimación de ingresos y estancias inadecuadas en un hospital comarcal. Rev Clin Esp 2000; 200: 654-8.

15. Gonzalez-Montalvo JI, Baztan JJ, Rodriguez-Manas L, San Cristobal E, Gato A, Ballesteros P, Salgado A. Ingreso hospitalario urgente: causas, justificación, factores sociales y sus diferencias en función de la edad. Med Clin (Barc) 1994; 103: 441-4.

16. Coast J, Peters T, Inglis A. Factors Associated with Inappropriate
Emergency Hospital Admisión in the UK. Int J Qual Health Care 1996; 8: 31-9.

17. Luis Zambrana Garcia J, Delgado Fernandez M, Cruz Caparros G, Martin D, Escalante M, Diez Garcia F, Ruiz Bailen M. Factores predictivos de estancias hospitalarias inadecuadas en un servicio de medicina interna. Med Clin (Barc) 2001; 117: 90-2.

18. Campbell J. Inappropriate admissions: thoughts of patients and referring doctors. J R Soc Med 2001; 94: 628-31.

19. Suarez Garcia F, Oterino de la Fuente D, Peiro S, Librero J, Barrero Raya C, et al. Factores asociados con el uso y adaptación de la hospitalización en personas mayores de 64 años de edad. Rev Esp Salud Publica. 2001; 75: 237-48.

20. Rodriguez-Vera J, de la Llave EP. Inappropriate admissions.J R Soc Med 2002 ; 95: 111

21. Panis LJ, Verheggen FW, Pop P. To stay or not to stay. The assessment of appropriate hospital stay: a Dutch report. Int J Qual Health Care. 2002; 14: 55-67

22. Martínez Fresneda M, Guerrero M, Cortés J, López MJ. Estudio de adecuación de estancias e ingresos en un hospital universitario mediante el empleo del Appropriateness Evaluation Protocol. Gestión Hospitalaria 2000; 11: 75-86.

23. San Roman Teran CM, Guil Garcia M, Fernandez Sepulveda S, Lorca Gomez J. Ingresos y estancias inadecuados en medicina interna. Med Clin (Barc) 2002; 118: 157.

24. Miron Canelo JA, Orosco Pedemonte JA, Alonso Sardon M, Saenz Gonzalez MC. Estimación de ingresos y estancias inadecuadas en un hospital comarcal. Rev Clin Esp 2000; 200: 654-8

25. Restuccia J. Manual de Utilización del Appropriateness Evaluation Protocol. Fundación Avedis Donabedian. Barcelona, 1995.

26. Fellin G, Apolone G, Tampieri A, Bevilacqua L, Meregalli G, Minella $\mathrm{C}$, et al. Appropriateness of hospital use: an overview of Italian studies. Int J Qual Health Care 1995; 7: 219-25.

27. Santos-Eggimann B, Paccaud F, Blanc T. Medical appropriateness of hospital utilization: an overview of the Swiss experience. Int J Qual Health Care 1995;7: 227-32.

28. Lang T, Liberati A, Tampieri A, Fellin G, Gonsalves Mda L, Lorenzo S, et al. A European version of the Appropriateness Evaluation Protocol. Goals and presentation. The BIOMED I Group on Appropriateness of Hospital Use. Int J Technol Assess Health Care 1999; 15: 185-97.

29. Bentes M, Gonsalves ML, Santos M, Pina E. Design and development of a utilization review program in Portugal. Int $\mathbf{J}$ Qual Health Care 1995; $7:$ : 201-12.

30. Lang T, Davido A, Logerot H, Meyer L. Appropriateness of admissions: the French experience. Int J Qual Health Care 1995; 7: 233-8.

31. Lombard I, Lahmek P, Diene E, Monnet E, Logerot H, Levy Soussan $\mathrm{M}$, et al. Cause of non-pertinent hospital stays: interobserver concordance using the French version of the Appropriateness Evaluation Protocol. Rev Epidemiol Sante Publique 2001; 49: 367-75. 